\title{
Acercamiento al Modelo Pedagógico Integrado para la enseñanza de hidrología subterránea
}

\section{Approach to Integrated Pedagogical Model for the teaching of groundwater hydrology}

\author{
María Fernanda Serrano-Guzmán ${ }^{1}$ \\ Directora General de Investigaciones y Docente titular \\ Facultad de Ingeniería Civil \\ Universidad Industrial de Santander \\ Santander, Colombia \\ mariaf.serrano@upb.edu.co \\ Diego Darío Pérez-Ruiz² \\ Director, Departamento de Ingeniería Civil e Industrial, \\ Facultad de Ingeniería, \\ Pontificia Universidad Javeriana, \\ Cali, Colombia \\ ddperez@javerianacali.edu.co \\ Guillermo Galvis-Hormiga ${ }^{3}$ \\ Especialista en Gerencia e Interventoría de Obras Civiles \\ Universidad Pontificia Bolivariana Seccional Bucaramanga \\ Santander, Colombia \\ guillermo_1035@hotmail.com
}

Recibido: 17 septiembre 2012 Aceptado: 13 marzo 2014 Corregido: 25 marzo 2014

Resumen: El sistema educativo de la Universidad Pontificia Bolivariana se ha apoyado de la investigación, básica y aplicada, para la implementación de un Modelo Pedagógico Integrado (MPI) en el que el discente es el responsable de su proceso de formación íntegral. El objetivo de este artículo es compartir las lecciones aprendidas en la experiencia de trabajo de investigación colectiva docentediscente producto de la cual se diseñó y construyó un modelo a escala reducida bidimensional de un acuífero con el cual el estudiante aborda la temática de la distribución del agua, y de manera específica, del uso del agua subterránea. El modelo a escala reducida del acuífero desarrollado, de dimensiones $1 \mathrm{~m} * 1 \mathrm{~m} * 0.10 \mathrm{~m}$, funciona a escala bidimensional incluyendo un acuífero confinado por una capa confinante (de arcilla) y un acuífero no confinado que finaliza con una superficie arenosa (expuesta a

$1 \quad$ Ingeniera Civil de la UIS Bucaramanga, Especialista en Gerencia de Proyectos de Construcción de la UIS Bucaramanga, Especialista en Ingeniería Ambiental de la Universidad Pontificia Bolivariana Seccional Bucaramanga, Magíster en Ingeniería en Ingeniería Civil de la Universidad de Puerto Rico, Doctorado en Ingeniería Civil de la Universidad de Puerto Rico. Desde el 2009 es la Directora General de Investigaciones de la Universidad Pontificia Bolivariana y es docente titular de la Facultad de Ingeniería Civil. Teléfono: 57-76-796220 Bucaramanga Colombia.

2 Ingeniero Civil de la Universidad del Cauca, Magíster en Ingeniería de Tránsito y Transporte de la Universidad del Cauca, Master en Ciencias en Ingeniería Civil de la Universidad de Puerto Rico, Doctorado en Ingeniería Civil de la Universidad de Texas en Arlington. Actualmente Profesor Asociado de la facultad de Ingeniería y Director del Departamento de Ingeniería Civil e Industrial, Facultad de Ingeniería, Pontificia Universidad Javeriana, Cali.

3 Ingeniero Civil y Especialista en Gerencia e Interventoría de Obras Civiles de la Universidad Pontificia Bolivariana Seccional Bucaramanga. Asesor y consultor. 
la atmósfera). La estructura y dimensiones del modelo a escala reducida permiten la planeación de actividades docentes para la transmisión de conocimiento sobre la hidráulica que rige la movilidad de fluidos en el suelo; la comprensión por parte de los estudiantes sobre la interrelación entre los niveles de agua subterránea y los de agua superficial y la simulación de derrames en la parte superficial, así como también la visualización de la movilidad de estos en la parte subterránea. Finalmente, este modelo a escala reducida se convierte en una estrategia pedagógica para la compresión de los procesos asociados a la hidrología subterránea.

Palabras claves: Acuífero, pedagogía, hidráulica, hidrología subterránea.

\begin{abstract}
Grounded on basic and applied research, the Universidad Pontificia Bolivariana system is working on the implementation of the Integrated Pedagogical Model (IPM) to help students develop their own academic training skills,. A collaborative effort between professors and students has been made to design and build a two-dimensional physical model of an aquifer at a small scale in order to understand the interaction of surface and groundwater resources. The physical model consists of a $1 \mathrm{~m} \times 1 \mathrm{~m} \times 0.1 \mathrm{~m}$ transparent box containing the two-dimensional system including an unconfined aquifer on top of an aquifer confined by a layer of clay. The dimensions of the physical model allow the professor and students to visualize the implications of phenomena such as groundwater flow, surface water and groundwater relationship, surface contaminant infiltration, fate and transport of ground water contaminants, and effect of ground water exploitation. In conclusion, small-scale physical models represent ideal pedagogical strategies which convey the potential of knowledge generation and better understanding of concepts and theories of the groundwater phenomenon.
\end{abstract}

Keywords: Aquifer, hydraulic, groundwater hydrology., pedagogy.

\title{
1.Introducción
}

El sistema nacional de Universidades de la Universidad Pontificia Bolivariana ha implementado el Modelo Pedagógico Integrado (MPI) con el cual se compromete al discente en su proceso formativo mediante el uso de la investigación como estrategia de aprendizaje. Esto implica un trabajo mayor por parte del estudiante quien se enfrenta a situaciones propias de su ejercicio profesional disciplinar (Salicetti y Romero, 2010).

Por tal razón, los lineamientos del MPI han motivado a los docentes a plantear diferentes tipos y métodos de investigación en el aula de clase como se evidencia en la Tabla 1. Para el caso específico del curso hidrología subterránea, pueden citarse los siguientes (Vargas, 2009): 


\section{Tabla 1}

Prácticas pedagógicas asociadas a métodos y/o tipos de investigación

\begin{tabular}{|c|c|}
\hline Tipo y/o métodos de investigación & Aplicación en el curso de aguas subterráneas \\
\hline $\begin{array}{l}\text { Visita de campo guiada por la autoridad ambiental competente. } \\
\text { Durante las visitas de campo se aplican las técnicas de la } \\
\text { encuesta o la entrevista en una población de estudio, con el fin } \\
\text { de identificar las necesidades o problemas que afectan un sector. }\end{array}$ & $\begin{array}{l}\text { - Para el desarrollo particular del curso adelantado se realizaron } \\
\text { visitas a comunidades en las que se evidenciaron situaciones } \\
\text { asociadas por la cantidad y/o por la calidad del agua y a } \\
\text { zonas en donde se encuentran los pozos de observación y de } \\
\text { explotación en Bucaramanga y su área metropolitana. }\end{array}$ \\
\hline $\begin{array}{l}\text { Consulta de reportes de caso. } \\
\text { Los estudios de caso corresponden al método de investigación que } \\
\text { se emplea como práctica regular para estudiar una comunidad } \\
\text { en particular. }\end{array}$ & $\begin{array}{l}\text { - Al respecto, se han revisado estudios realizados por la } \\
\text { autoridad competente sobre sectores que se abastecen de } \\
\text { depósitos subterráneos. Como complemento a la consulta, } \\
\text { se han hecho discusiones sobre el uso de este recurso que en } \\
\text { ocasiones es explotado de manera clandestina por parte de } \\
\text { los negocios de lavado de autos. }\end{array}$ \\
\hline $\begin{array}{l}\text { - Investigación-acción. } \\
\text { Mediante la investigación-acción se intentan estudiar los } \\
\text { problemas científicamente para evaluar las decisiones y } \\
\text { acciones. }\end{array}$ & $\begin{array}{l}\text { El uso del modelo a escala reducida permite reflexionar sobre } \\
\text { la práctica y la postura epistemológica y teórica sobre temas } \\
\text { particulares de la explotación del recurso subterráneo y la } \\
\text { movilidad del fluido cuando esta se da. }\end{array}$ \\
\hline $\begin{array}{l}\text { - Investigación participativa. } \\
\text { La investigación participativa se fundamenta en identificar } \\
\text { necesidades propias de un colectivo. }\end{array}$ & $\begin{array}{l}\text { - Al respecto, lo referente al manejo de disponibilidad de agua } \\
\text { potable en zonas áridas del departamento se orientaron los } \\
\text { trabajos hacia la definición de alternativas de suministro de } \\
\text { agua para estos casos. }\end{array}$ \\
\hline $\begin{array}{l}\text { - Investigación aplicada. } \\
\text { También conocida como investigación práctica o empírica, se } \\
\text { caracteriza porque se utilizan los conocimientos adquiridos y se } \\
\text { adquieren otros, después de implementar la práctica basada en } \\
\text { investigación, con la cual se resuelven problemas de la vida cotidiana. }\end{array}$ & $\begin{array}{l}\text { - Para el caso del curso de aguas subterráneas, durante } \\
\text { las visitas de campo se tomaron datos particulares para } \\
\text { reproducir un modelo matemático que sustentara las } \\
\text { decisiones teóricas planteadas. }\end{array}$ \\
\hline
\end{tabular}

Como se menciona previamente, el MPI permite el uso de diferentes tipos de investigación o métodos aplicados favoreciendo el aprendizaje basado en problemas, lo cual implica el trabajo cooperativo y la enseñanza centrada en el estudiante (Serrano-Guzmán, Solarte-Vanegas, Pérez-Ruiz y Pérez-Ruiz, 2011).

Este artículo presenta un resultado del trabajo colaborativo entre docente-discente para la implementación del MPI en el curso de aguas subterráneas, que conllevó al diseño y construcción de un modelo a escala reducida de acuífero con el cual se facilita el proceso enseñanza-aprendizaje de fenómenos de movilidad del agua, la interacción entre el medio superficial y subterráneo y se busca que el discente adquiera las competencias, a escala laboratorio, con la expectativa que puedan ser extrapoladas a escala real, es decir en el trabajo de campo.

El nacimiento de la psicología cognitiva hacia los sesenta ofreció un paradigma conceptual para describir el diseño, el cual era considerado como una actividad mental invisible. Los estudios iniciales se basaron en la solución de problemas de las ciencias cognitivas y la inteligencia artificial. Poco a poco, el diseño fue considerado como un tipo de solución de problemas con lo cual se buscaba el procedimiento más satisfactorio, algo así como armar un rompecabezas partiendo de una serie de etapas, cada una de las cuelas con funciones diferentes (Eastman, Mc Cracken y Newstetter, 2001). Para la construcción del modelo a escala reducida, por tanto, resulta imperioso considerar el material del cual se construye y verificar si el diseño concebido permite determinar los parámetros de medición para el cual fue concebido. 
Un diseño de un modelo a escala reducida puede llegar a ser bueno, en cuanto se considere la visión de dicho modelo desde diferentes perspectivas, permita la generación de alternativas de uso y el planteamiento de un funcionamiento en condiciones extremas, de manera que se permita un progreso educativo (Eastman, Mc Cracken y Newstetter, 2001), en el que se tenga en cuenta la forma como el educando aprende, aplica e integra el conocimiento recibido con el modelo a escala reducida para la solución de un problema específico en el contexto de su desempeño laboral. El educando y futuro profesional, tienen entonces una serie de información aprendida realmente, otra que ha construido haciendo, otra con relación a sus habilidades y destrezas personales; y otra, que es por la experiencia propia que responde muchas veces a sus necesidades (Constantinides, Montalvo y Silverman, 2013).

Un punto de partida, es la elaboración de esquemas (bocetos) que permiten visualizar un comportamiento dado. Para el caso específico del acuífero, durante la participación de los estudiantes en las discusiones sobre el diseño final al ser construido se observó la integración de los conceptos teóricos y el uso de la memoria para correlacionar las experiencias de las visitas de campo en las zonas de explotación de agua mediante el sistema de pozos de bombeo. Adicionalmente, se revisaron los resultados de modelos de Putranto y Chen (2013) y los estudios de Chen, Huan y Chakma (2000) y Kechavarzi, Soga e Illangasekare (2005) los cuales han sido de utilidad para simular la movilidad de compuestos en el campo.

\section{Imágenes mentales y la estructura del conocimiento}

La representación mental del conocimiento ha sido tema de estudio desde Platón y abarca una serie de doctrinas sicológicas desde aquellas que desconocen las imágenes mentales hasta aquellas que consideran una estructura simbólica proposicional más compleja. Eastman, Mc Cracken y Newstetter (2001) mencionan que existe la habilidad de aprendizaje, la cual estaba influenciada por la habilidad mental de visualizar la información de referentes previos. Estos conocimientos previos o preconceptos son utilizados, algunas veces, de manera no organizada pudiendo con ello, posteriormente interpretar eventos.

El éxito en la asociación de los preconceptos o presaberes depende de la habilidad de los individuos y también está asociado con el entrenamiento que haya recibido la persona, de manera que la estructura del conocimiento se haga mediante el uso de sensaciones y de la memoria, siendo la selectiva y a largo plazo aquella que juega un papel importante en el proceso de enseñanza aprendizaje, es decir, al momento de aprender a aprehender (Eastman, Mc Cracken y Newstetter, 2001). En ocasiones, las asociaciones temporales o espaciales permiten también el aprendizaje. Para la conceptualización del modelo a escala reducida del acuífero cumplieron un papel importante las experiencias de campo de los estudiantes que colaboraron con el diseño. 


\section{Hacia la construcción de conceptos partiendo de imágenes mentales de un sistema de hidrología subterránea}

La contaminación de suelos y aguas subterráneas por compuestos (contaminantes) está causando deterioro a la calidad del ambiente y pone en riesgo la salud humana. Estos contaminantes pueden permanecer por largo tiempo en el ambiente (Serrano-Guzmán, Pérez Ruiz, Puppala y Padilla, 2010) e interactúan en el medio poroso a través de las diferentes fases (Marinack Jr., Mpagazehe y Higgs III, 2012). Su movilidad hace difícil la localización, caracterización y posterior remediación de los suelos en donde se han encontrado áreas expuestas a este tipo de contaminación. Teniendo en cuenta que este conocimiento es complejo de transferir en el aula de clase, se acordó establecer mediante una estrategia pedagógica, el diseño y construcción de un modelo a escala reducida con el cual se pudieran observar, además de la movilidad del fluido, los procesos de transporte asociados a los acuíferos que contienen el preciado líquido.

El diseño y construcción del acuífero implicó la intervención del docente y los discentes que de manera conjunta tomaron decisiones sobre el material, las dimensiones y las especificaciones para que finalmente con este modelo a escala reducida fuera posible el desarrollo de prácticas de explotación de agua subterránea en acuíferos confinados y no confinados y la verificación de la movilidad de una contaminación simulada. Las etapas relevantes para la construcción se describen a continuación:

- Aprobación del diseño del tanque. Para ello se revisaron las dimensiones de los modelo a escala reducidas a escala bidimensional que otros investigadores habían desarrollado en estudios previos. El equipo de trabajo acordó hacer uso de un modelo a escala reducida en vidrio de dimensiones efectivas para confinamiento del material granular de $100 \mathrm{~cm} * 100 \mathrm{~cm} * 10 \mathrm{~cm}$, con compartimientos laterales para el ingreso del agua y la movilidad de ésta hacia el.

- Representación de un sistema confinado. Con el fin de ejemplificar el acuífero confinado según los presaberes y los conceptos de clase, se decidió rellenar el tanque hasta una altura que permitiera la instalación de dos pozos (uno de observación y otro de explotación). La capa confinante se preparó con bentonita.

- Representación de un sistema no confinado. Con el fin de ejemplificar el acuífero no confinado según los presaberes y los conceptos de clase, se decidió rellenar el tanque hasta la superficie, dejando una capa que se recubrió con un lecho rocoso para simular un río o lago. Sobre este elemento hidráulico se simuló un derrame superficial. Adicionalmente, en el tramo no confinado se dejó instalada una manguera para simular un derrame de un elemento enterrado en el subsuelo. (Figura 1). 


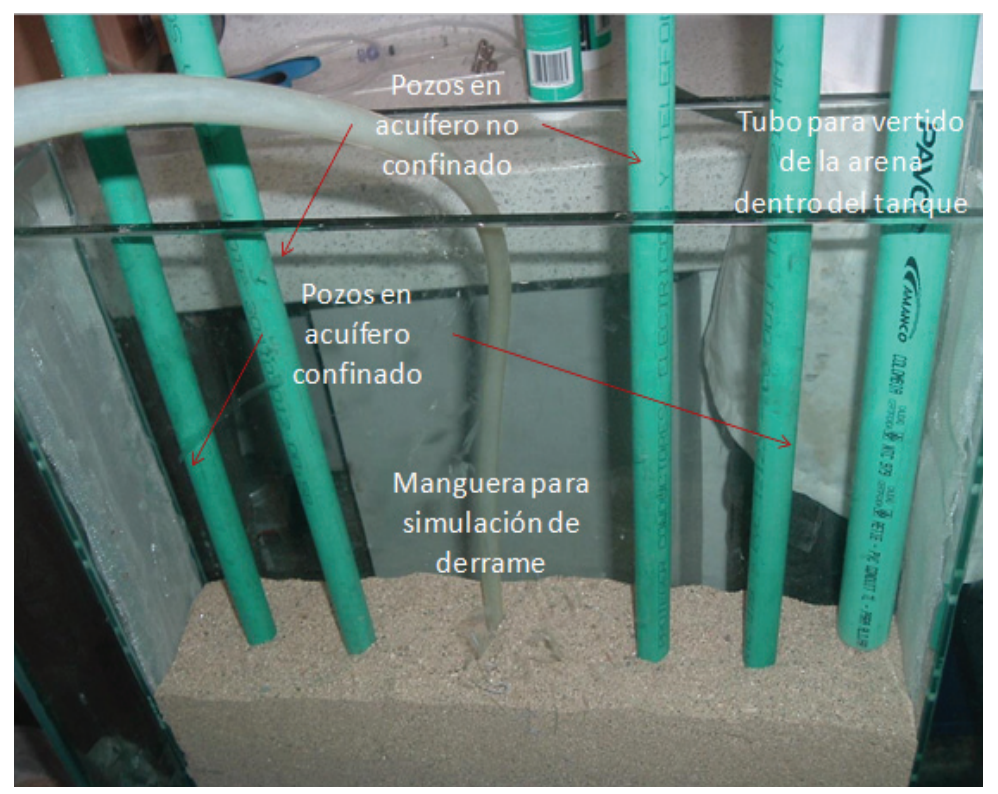

Figura 1. Construcción de los depósitos confinado y no confinado de cada acuífero.

Sistemas de control de flujo. Teniendo en cuenta que el modelo a escala reducida debería permitir la simulación de condiciones de explotación de agua del sistema, se dejó instalado un sistema de salida de agua manual, el cual se preparó con mangueras de macrogoteo y otro mecánico, haciendo uso de una bomba. Adicionalmente, la instalación de la manguera permitió la simulación del derrame subterráneo (Figura 2).

\section{Experiencias significativas}

El modelo a escala reducida desarrollado se trabajó en conjunto con los discentes y el docente del curso permitiendo la construcción de una herramienta que facilitó el proceso de enseñanza por parte del docente y de aprendizaje por parte de los discentes quienes a lo largo del curso debieron documentarse, revisar el estado del arte sobre el tema, familiarizarse con las fórmulas y conceptos asociados con la hidráulica de pozos y llevar a cabo experimentos a escala de laboratorio que posteriormente validaron en campo.

Durante el desarrollo del curso y la correspondiente construcción del modelo a escala reducida se observó que los estudiantes adquirieron competencias profesionales que se

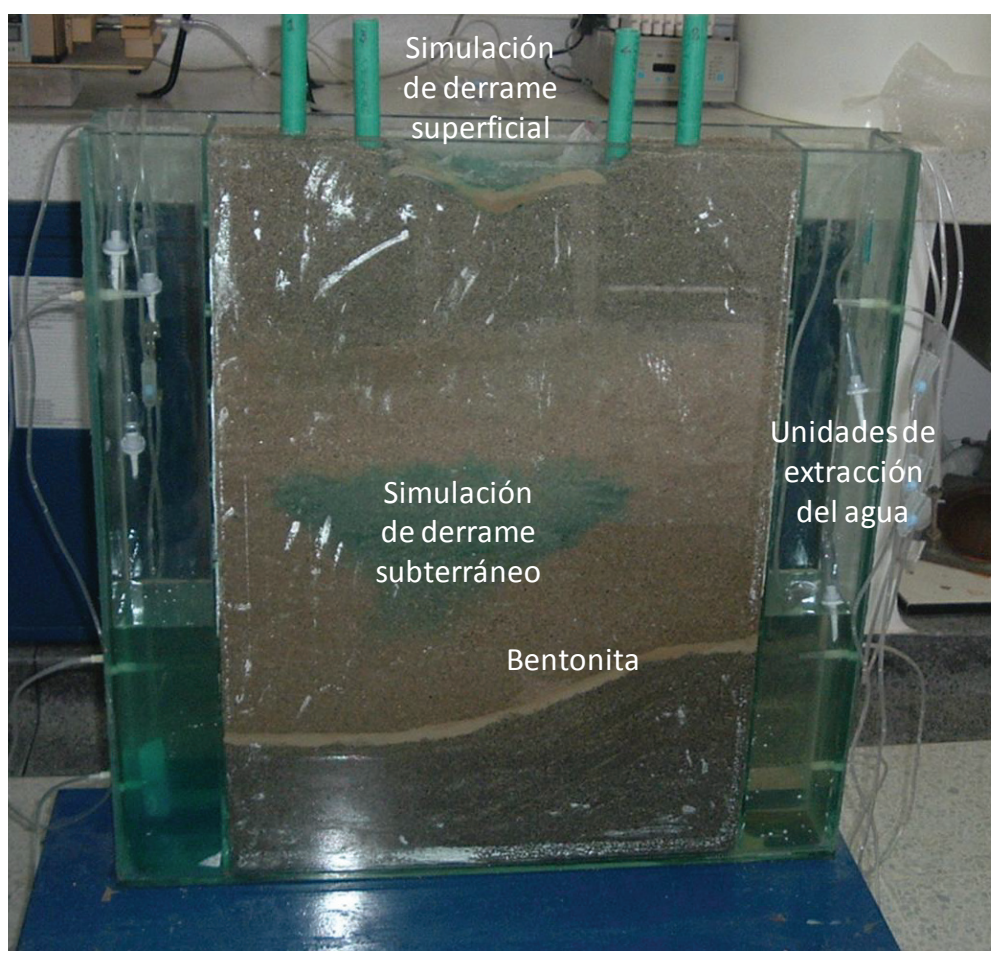

Figura 2. Simulación de un derrame. complementaron con su desarrollo social y su habilidad comunicativa oral y escrita. Se resumen a continuación los esquemas de evaluación utilizados (Tabla 2). 


\section{Tabla 2}

Instrumentos asociados a la evaluación de competencias

\begin{tabular}{|c|c|c|}
\hline La competencia implica & $\begin{array}{c}\text { Consecuencias para la enseñanza } \\
\text { apredizaje y la evaluación }\end{array}$ & Instrumento utilizado \\
\hline $\begin{array}{l}\text { Integrar conocimientos asociados } \\
\text { a la hidrología subterránea. }\end{array}$ & $\begin{array}{l}\text { Relacionar los conocimientos con las } \\
\text { condiciones del entorno. }\end{array}$ & $\begin{array}{l}\text { Proyecto de clase asociado con el } \\
\text { análisis de un caso de estudio. }\end{array}$ \\
\hline Realizar prueba de pozo. & $\begin{array}{l}\text { Organizar el recurso humano y el } \\
\text { equipo para la correcta ejecución de } \\
\text { las pruebas }\end{array}$ & $\begin{array}{l}\text { Selección de los métodos de captura } \\
\text { de datos. }\end{array}$ \\
\hline Actuar de forma contextual & $\begin{array}{l}\text { Identificar la tendencia de movilidad } \\
\text { de un contaminante dependiendo de } \\
\text { la localización en los acuíferos. }\end{array}$ & $\begin{array}{l}\text { Simulaciones desarrolladas con } \\
\text { prototipo. }\end{array}$ \\
\hline Entender de forma dinámica. & $\begin{array}{l}\text { Evaluar la variación de los niveles } \\
\text { freáticoscuando se realiza la } \\
\text { explotación de un pozo. }\end{array}$ & $\begin{array}{l}\text { Seguimiento de los niveles de agua } \\
\text { verificación de los modelos teóricos. }\end{array}$ \\
\hline Actuar con autonomía & $\begin{array}{l}\text { Evaluar la capacidad de autoreflexión } \\
\text { del estudiante sobre el caudal a } \\
\text { bombear en un pozo. }\end{array}$ & $\begin{array}{l}\text { Seguimiento a la determinación de } \\
\text { ecuación de eficiencia de pozo. }\end{array}$ \\
\hline
\end{tabular}

Según Cano-García (2008) estas implicaciones se pueden analizar de manera separada así:

1. La integración de conocimientos se hace mediante los trabajos de campo y las prácticas de laboratorio. Justamente, es durante estos ejercicios que se generan también los roles que a futuro desempeñarán los profesionales.

2. La prueba de pozo se evalúa durante la ejecución, el proceso y el resultado final, realzando la importancia de los métodos de captura. En este caso, es relevante la organización del equipo de trabajo.

3. La identificación de la tendencia de movilidad implica que se resuelvan las simulaciones en diferentes contextos. Por tal razón, se dejaron en el sistema, un sistema superficial (el lago o río) y un sistema subterráneo (la manguera enterrada).

4. La verificación de los niveles de agua en los distintos pozos permite la comprensión del efecto de la explotación del recurso subterráneo. Adicionalmente, se permite la aplicación de los modelos teóricos revisados durante las sesiones teóricas del curso. El modelo a escala reducida permite modificar los niveles y revisar diferentes comportamientos de la hidráulica de pozos.

5. Cada actividad desarrollada se hace respetando la autonomía del grupo de trabajo. El apoyo del docente para que se mantenga el equipo trabajando en forma coherente es relevante. En la actividad que más se evidenció el trabajo autónomo fue durante la verificación de la ecuación de eficiencia de un pozo, que implicó un trabajo de laboratorio considerando condiciones llevadas a campo. 


\section{Conclusiones}

El modelo a escala reducida de acuífero diseñado y construido surgió como una iniciativa del semillero de investigación SIIC y del grupo de Investigación Decor, propiciando una herramienta para llevar el modelo pedagógico integrado dentro del curso de Hidrología Subterránea. Se observa que con este acuífero es posible realizar además de estudios de caso, prácticas variadas que pueden apoyarse en el trabajo colaborativo para la validación de resultados obtenidos utilizando software especializado.

En este caso, como las paredes del modelo a escala reducida del acuífero eran de vidrio, se permitió seguir la movilidad de los fluidos mediante el uso de un trazador. En cuanto al trazador, se recomienda utilizar aquel que no afecte de manera permanente las paredes del vidrio por cuanto limitaría la repetitividad de los experimentos.

Para el desarrollo de este montaje se utilizaron materiales conseguidos en el medio universitario y que fueron adaptados para los fines del estudio. Es así como: los filtros fueron adaptados utilizando tela (organza); las unidades de macrogoteo para controlar los niveles de agua; la "contaminación" simulada con tinte de culinaria; los sistemas de bombeo de pozos adaptados para ser ejecutados de manera manual; el sistema de drenaje del tanque desarrollado aplicando mecánica de fluidos, entre otros aspectos.

\section{Agradecimientos}

A la Dirección General de Investigaciones y a Ecopetrol-ICP por el apoyo financiero para la realización del acuífero. A los estudiantes del semillero de investigación en Ingeniería Civil, principalmente a Leonardo Almeyda, Camilo Mantilla, William Quintana, Lizbeth Hernández, Nazly Galindo, Diego Duarte e Iván Prieto.

\section{Referencias Bibliográficas}

Cano-García, M.E. (2008). La evaluación por competencias en la educación superior. Profesorado. Revista de currículum y formación del profesorado, 12 (3), 1-16.

Chen, Z. G.H. Huang y A. Chakma (2000), Risk assessment of a petroleum-contaminated site through a multiphase and multi-component modelling approach. [Evaluación de riesgos de sitios contaminados por petróleo a través de aproximaciones de modelos multifase y multicomponentes], Journal of Petroleum Science and Engineering, 26, 273-281.

Constantinides, P., R. Montalvo, y S. Silverman. (2013). Teaching processes in elementary physical education clases taught by specialist and nonspecialist. [Procesos de enseñanza en clases de educación física elemental a cargo de especialistas y no especialistas]. Teaching and Teacher Education, 36, 68-76. 
Eastman, C. y W. M. Mc Cracken y W. Newstetter. (2001). Design Knowing and Learning: Cognition in Design Education. [Diseñando conocimiento y aprendizaje: Cognición en Educación de Diseño]., Chapter 8, New Directions in Design.

Kechavarzi, C., K. Soga y T.Illangasekare (2005). Two dimensional laboratory simulation of LNAPL infiltration and redistribution in the vadose zone. [Simulación en dos dimensiones a escala laboratorio de la infiltración y redistribución de la zona vadosa], Journal of Contaminant Hydrology, 76, 211-233.

Marinack Jr., M.C. y J. N. Mpagazehe y C. F. Higgs III (2012). An Eulerian, lattice-based cellular automata approach for modelling multiphase flows. [Un enfoque euloriano basado en una aproximación celular de flujos multifase], Powder Technology, 221, 47-56.

Putranto, A. y Chen, X. D. (2013). Multiphase modelling of intermittent drying using the spatial reaction engineering approach (S-REA). [Modelación multifase del secado intermitente usando la reacción de aproximación espacial S-REA], Chemical Engineering and Processing, 70, 169-183.

Salicetti Fonseca A. y Romero Cerezo, C. (2010). La plataforma de apoyo a la docencia como opción metodológica para el aprendizaje de competencias. Revista Educación, 34 (1), 83-100.

Serrano-Guzmán, M.F.; Pérez Ruiz, D.D; Puppala, A y Padilla. I. (2010). CWR: Alternativa para detección de NAPLs. Puente Revista Científica, 5 (2), 25-35.

Serrano-Guzmán, M.F., Solarte-Vanegas, N. C.; Pérez-Ruíz, D. D. y Pérez -Ruiz. A. (2011). La investigación como estrategia pedagógica del proceso de aprendizaje para ingeniería civil. Revista Educación, 35 (2), 1-33.

Vargas Cordero, Z. R. (2009).La investigación aplicada: una forma de conocer las realidades con evidencia científica. Revista Educación, 33(1), 155-165. 